\title{
Revisión narrativa y desarrollo de un programa de intervención para la disminución de los efectos del Alzheimer a través de la práctica del Surf en Personas Mayores Narrative review and development of an intervention program for reducing Alzheimer's effects through the practice of Surf in older people \\ Mateo Real Pérez, Cristina Robles Rodríguez, Jesús Gustavo Ponce González \\ Universidad de Cádiz (España)
}

\begin{abstract}
Resumen: La enfermedad de Alzheimer (EA) y otros trastornos demenciales son factores importantes a tener en cuenta en los índices de morbilidad $\mathrm{y}$ mortalidad en la sociedad actual. El deterioro cognitivo tiene un impacto negativo en las actividades funcionales de los pacientes con EA, y la eficacia del tratamiento farmacológico es limitada y no consigue detener el deterioro cognitivo asociado a esta demencia. Por lo que el objetivo principal de este artículo es revisar la bibliografía y diseñar estrategias de intervención para retrasar la aparición o reducir el riesgo de padecer alteraciones neurodegenerativas provenientes de la EA. En la última década ha aumentado el interés hacia el diseño y la experimentación a partir de nuevas terapias no farmacológicas. El ejercicio físico es un tipo de terapia complementaria en el que la práctica habitual tiene un efecto beneficioso para la salud en la EA. Sus efectos se circunscriben a nivel físico y funcional mejorando el mantenimiento de la autonomía y la disminución de la carga de los cuidadores. En este artículo se hace una revisión de los beneficios del ejercicio sobre la EA, proponiendo un programa de intervención de ejercicios multitarea y de mejora del acondicionamiento físico, utilizando el medio acuático a través del surf, para prevenir la aparición de EA. Se estima que los sujetos obtengan mejoras en el rendimiento cognitivo, el equilibrio y la autonomía personal en las tareas de la vida diaria. Además, estimamos que el programa de entrenamiento implicará un incremento en algunos parámetros de condición física, como la capacidad aeróbica.
\end{abstract}

Palabras clave: Alzheimer; Surf; Ejercicio; Funciones Cognitivas; Personas Mayores; Calidad de Vida

\begin{abstract}
Alzheimer's disease (AD) and other dementia disorders are important factors of morbidity and mortality in our society. Cognitive impairment has a negative impact on the functional activities of AD patients, and the effectiveness of drug treatment is limited and fails to avoid cognitive decline associated with this dementia. Hence, the main aim of this work is to review the literature, and to design an intervention to reduce the risk of neurodegenerative disorders provoked by AD. There has been an increased interest in designing and testing new non-drug therapies during the last decade. Exercising is a type of complementary therapy that has a beneficial effect on health in persons with AD. The main benefits of exercising are focused on physical and functional improvement, which increase the maintenance of autonomy and decrease caregivers' burden. This study reviews the benefits of exercising on patients with $\mathrm{AD}$, and proposes an intervention program to prevent the onset of $\mathrm{AD}$ based on multitasking training by means of improving physical fitness through surfing. It is deemed that patients could improve their cognitive performance, balance, and personal autonomy in everyday life tasks. Also, we consider that the proposed training program should determine an increase in some parameters of physical fitness, such as aerobic capacity.
\end{abstract}

Keywords: Alzheimer; Surfing; Exercising; Cognitive Functions; Older People, Quality of Life.

\section{Introducción}

La enfermedad del Alzheimer(EA) ha incrementado en las últimas décadas, tal y cómo se refleja en el artículo: «Reeducación Psicomotriz en personas con enfermedad de Alzheimer» (Serdá I Ferrer; 2013). Aproximadamente 44 millones de personas sufren demencia en todo el mundo en la actualidad, estimando que en el año 2030, 66 millones de personas podrían padecer algún trastorno neurodegenerativo. España se encuentra entre los cinco países europeos que presenta más casos de Alzheimer con una prevalencia de 1,36\% con más de 800.000 personas con Alzheimer, ligeramente superior a la media europea, de 1,27\%. Además, cabe destacar que los cuidados del Alzheimer en España suponen 24.000 millones de euros anuales, según datos de la Confederación Española de Asociaciones de Familias de Personas con Alzheimer y otras demencias (CEAFA).

LaEA secaracterizaporuna pérdidainsidiosa dela facultad cognitiva, de las alteraciones conductuales anímicas y psiquiátricas que generan un estado de dependencia. Un diagnóstico de la enfermedad de Alzheimer les cambia la vida no solo a la persona con la enfermedad, sino también a sus familiares y amigos.

\section{Características de la enfermedad del Alzheimer}

Entre las facultades cognitivas afectadas destacamos la pérdida temprana de la capacidad de fijar nueva información y recordar acontecimientos (amnesia); reconocer los objetos en su entorno (agnosia); comunicarse, expresarse y entender (afasia). Asu vez, existe una pérdida progresiva de la capacidad física y funcional en personas con EA, impidiendo la realización de las actividades de la vida cotidiana (apraxia).

Fecha recepción: 19-01-16. Fecha de aceptación: 27-11-16 Jesús Gustavo Ponce González

jesusgustavo.ponce@uca.es
Garuffi et al. (2013); indica que el EA, se caracteriza por el deterioro cognitivo progresivo y la disminución de la funcionalidad. Esto da lugar a que los pacientes tengan dificultades, o incluso les sea imposible llevar a cabo las acciones y actividades de la vida cotidiana.

Tratamiento farmacológico para la enfermedad deAlzheimer

La enfermedad de Alzheimer es una patología de evolución lenta. Desde que aparecen los primeros síntomas hasta que se inicia una etapa de mayor gravedad pueden pasar entre 5 y 20 años, dependiendo de cada persona. Por el momento no existeningún tratamiento que revierta el proceso de degeneración que comporta esta enfermedad. Sin embargo, sí se dispone de algunos fármacos que pueden retrasar, en determinadas etapas de la enfermedad la progresión de la patología.

Se utilizan los anticolinesterásicos o inhibidores de la acetilcolinesterasa, fármacos que elevan los niveles de acetilcolina en el cerebro. Galantamina, donepezilo y rivastigmina son los fármacos indicados en las primeras etapas de la enfermedad (Montón et al., 2011). Con estos medicamentos se mejoran las fases iniciales y moderadas de la patología, retrasando el deterioro de la memoria y la atención. Este tipo de tratamiento se combina con otro sintomático, que se administra, a medida que el paciente va denotando diversos síntomas que acompañan al mal de Alzheimer, tales como la depresión, estados de agitación, alteraciones del sueño, o complicaciones más tardías como incontinencia de esfínteres, estreñimiento, infecciones urinarias, úlceras provocadas por la inmovilidad o tromboflebitis.

Por otro lado, la vacuna,AN-1792, se basa en una forma sintética de la proteína beta-amiloide, proteína que conforma las placas en los cerebros de los pacientes con Alzheimer y estimula al sistema inmunológico para eliminar las placas ya formadas y evitar la aparición de otras nuevas.

Sin embargo, la eficacia del tratamiento farmacológico es limitada y no consigue detener el deterioro cognitivo asociado a las demencias (Serdá I Ferrer; 2013). Por este motivo, ha aumentado el interés hacia el 
diseño y experimentación a partir de nuevas modalidades de tratamiento no farmacológicas. Recientemente, se ha planteado el ejercicio físico como herramienta terapéutica complementaria donde la práctica habitual supone tener un efecto beneficioso para la salud en la EA (Burge, E. et al.; 2012). Sus efectos se circunscriben a nivel físico y funcional mejorando el mantenimiento de la autonomía, la disminución de la carga de los cuidadores y el riesgo de institucionalización (Serdá I Ferrer; 2013).

\section{Actividad Física y Alzheimer}

El Alzheimer también provoca una pérdida de capacidad física y funcional del sujeto, que se ve agravado en los casos dondeno existe una intervención para prevenir la pérdida insidiosa de facultades cognitivas, disminuyendo de manera significativa la calidad de vida de los pacientes con este tipo de demencia.

Es esencial el diagnóstico precoz de EA ya que, a medida que avanza, se hace más difícil frenar el deterioro producido por la muerte de las células nerviosas y la pérdida de tejido en todo el cerebro. Así mismo, los estudios realizados con programas de intervención basados en la actividad física se centran en aquellos pacientes que se sitúan en la fase temprana o moderada de la enfermedad. Estudios realizados por Serdá I Ferrer et al. (2013) han encontrado mejoras significativas en la capacidad física y la calidad de vida en pacientes con EA que se han sometido a un programa de entrenamiento basado en tareas psicomotrices. De Andrade et al. (2013) y Cohelo et al. (2012) demostraron que con un programa multimodal de 4 meses que incluye ejercicios de doble tarea combinado con ejercicio físico, estimulando el equilibrio y la capacidad cognitiva, se obtenían mejoras en las actividades de doble tarea, el control postural, el equilibrio y la capacidad funcional y cognitiva de los sujetos con EA.

Por otro lado, investigaciones centradas en indagar sobre correlación entre ejercicios aeróbicos y Alzheimer; como las llevadas a cabo por: Baker, L. et al. (2010), Garuffi, M. (2012), Stella, F. et al. (2012); mostraron mejoras asociadas al entrenamiento de resistencia cardiorrespiratoria en los grupos de entrenamiento con EA temprano y moderado. Además, se hallaron muestras significativas demostrando que el ejercicio aeróbico puede convertirse en un modelo terapéutico alternativo en las personas mayores con Alzheimer (Fang, Y. et al. 2012), favoreciendo el mantenimiento de las funciones cognitivas, el equilibrio y la agilidad, sin aumentar el riesgo de caída (Hernández, S.; 2010), reduciendo el deterioro de la capacidad funcional (Canonici,A.; 2012).

\section{Medio acuático como herramienta terapéutica}

Existen investigaciones que demuestran la eficacia de programas de ejercicios basados en la hidroterapia y han sido reconocidos como un medio que favorece el desarrollo de la condición física y motora, las habilidades sociales y la autoestima en personas con discapacidad (Chrysagis, Douka, Nikopoulos, Apostolopoulou \& Koutsouki., 2009; Conatser, P., 2007; Maniu., Maniu \& Benga, 2013). Los efectos beneficiosos de la hidroterapia para la salud y más concretamente del surf recreacional, combinados con una mejora de la condición física propia del deporte, podría mejorar la calidad de vida de las personas con EA.

En efecto, los estudios realizados por Armitano (2013) y Clapham, Armitano, Lamont, Audette (2014) han demostrado que un programa de surf adaptado mejora la condición física general, con una mejora en pacientes con otras enfermedades que alteran psicológicamente las áreas del cerebro, tales como el autismo.

Otros autores, como Fleischmann, D. (2011)han demostrado la eficacia del surf para estimular la función vestibular, relacionado con el equilibrio y la percepción espacial, en un sujeto con amputaciones y daños por traumatismo cerebral. Además, este deporte implica movimientos de los grandes grupos musculares, lo cual puede aumentar las concentraciones de serotonina, noradrenalina y dopamina, neurotransmisores del sistema nervioso que pueden estar afectados y disminuidos por la enfermedad deAlzheimer (Fleischmann, D., 2011). Esto se tradujo en mejoras en el equilibrio y disminución en el grado de depresión que presentaba, uno de los problemas que también afecta a las personas con EA.

Los beneficios en los síntomas psicológicos puede deberse a que se combina dos tipos de modalidades utilizadas en la actualidad para la mejora de la salud mental: la terapia de grupo y el ejercicio físico. Las diferentes asociaciones de surf, utilizan este tipo de actividades en formato de grupo en poblaciones especiales, favoreciendo la cohesión entre los participantes, resultando ser mucho más gratificante al compartir el bienestar por la práctica de ejercicio físico y los progresos en esta modalidad deportiva con los demás compañeros.

Estos resultados demuestran que esta modalidad podría tener una transferencia positiva en los procesos mentales de los sujetos, aumentando la estimulación cognitiva y disminuyendo el deterioro de facultades en personas con EA. Además, ofrece la oportunidad de llevar a cabo una participación deportiva independiente, sin reglas complicadas o contacto cercano con otros, que ayuda a potenciar las habilidades físicas y a superar las barreras sociales de los sujetos (Delaney y Madigan, 2009, citado por Clapham, 2014).

Por otro lado, los beneficios demostrados de la hidroterapia sobre personas con discapacidad y trastornos neuronales (Chrysagis et al., 2009; Conatser, P., 2007; Maniu et al., 2013), nos hace plantear el surf como una herramienta terapéutica para la población con EA en fase temprana.

Si nos centramos en la implicación de las personas mayores en la práctica del surf, también encontramos estudios realizados con surfistas veteranos que demuestran, no solo que las personas con más de 55 años pueden surfear, si no que muestran un mejor control neuromuscular y un mejor mantenimiento de la postura, lo que influye positivamente al equilibrio (Zhou, Martin, Bezerra \& Crowley, 2008).

Centrándonos básicamente en el deporte, como bien indica John Barlow (2013), el surf implica diferentes aspectos físicos tales como la agilidad y la fuerza explosiva. Surfear una ola implica una movilización de los segmentos corporales con cambios de dirección a diferentes velocidades en constante cambio. El cuerpo se ve envuelto en un entorno de incertidumbre, implicando un importante factor de percepción espacio-temporal, donde todo depende de las habilidades abiertas del sujeto. Esto se traduce en trabajo físico y cognitivo importante, de reconocimiento y reacción ante los diferentes estímulos para responder eficazmente ajustando la ejecución técnica de los movimientos.

Pero lo más importante para plantearnos elaborar el siguiente programa de intervención es la transferencia que tiene en el trabajo de la capacidad aeróbica en el medio acuático. Un estudio elaborado con análisis de vídeo por Meier et al. (1991) proporciona información útil sobre las demandas físicas del deporte, observando la actividad física durante una hora de surf recreativo. Se clasificaron los movimientos en cuatro categorías para determinar que el máximo tiempo empleado durante la actividad está destinado a la remada en posición prono (44\%), por delante del tiempo de espera en equilibrio sentado sobre la tabla (35\%) y otras actividades como hacer el pato (16\%). El tiempo empleado en surfear la ola únicamente ocupa un $5 \%$ del total de la actividad recreacional; por lo tanto, lo más interesante es la implicación en el sistema cardiorrespiratorio que tiene la actividad haciendo referencia al tiempo de remada.

La implicación del sistema aeróbico, el equilibrio y las habilidades coordinativas en la actividad, así como el importante factor cognitivo de percepción espacio-temporal y reconocimiento de los estímulos [Figura 1], nos muestra lo beneficioso que puede llegar a ser respecto a la prevención y el tratamiento auxiliar de las enfermedades neurodegenerativas como el Alzheimer en sus fases más tempranas en personas mayores.

Por todo lo expuesto, nos planteamos diseñar un programa de intervención con personas con EA en fase leve, que incluyera distintos tipos de ejercicio basándonos en tres pilares básicos para el trabajo de la condición física en esta población especial: i) las actividades de doble 


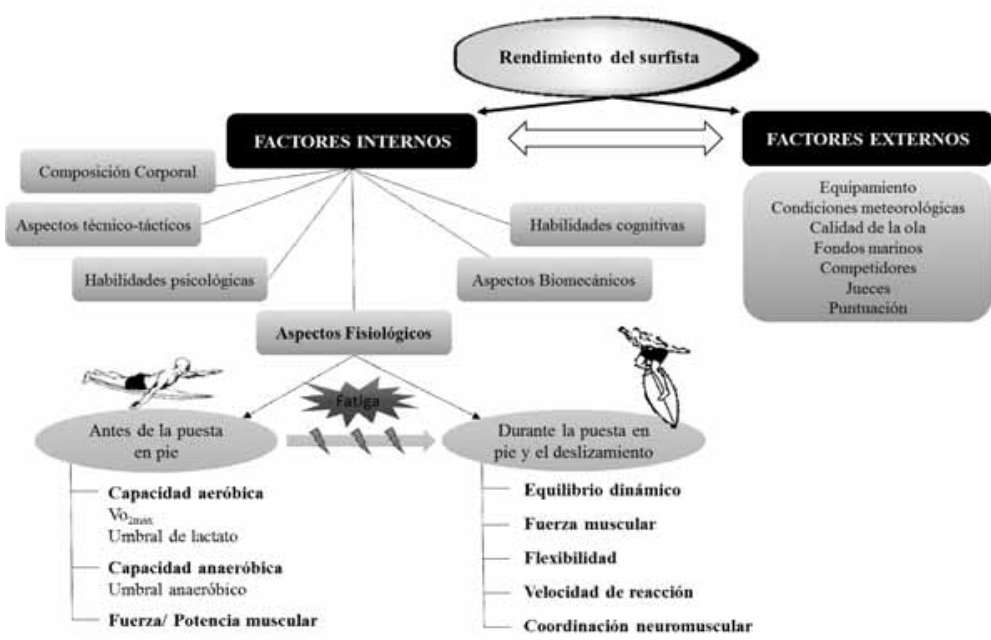

Figura 1. Aspectos fisiológicos y cualidades físicas relevantes durante la práctica del surf (Adaptado de Méndez-Villanueva et al., 2005).

tarea, para estimular la capacidad cognitiva (DeAndrade, L. et al., 2013; Cohelo, F. etal., 2012); ii)el equilibrio, para prevenir el riesgo de caída en mayores (Hernández, S. et al., 2010); y iii) el surf, como medio terapéutico para mejorar la capacidad aeróbica, equilibrio, capacidad de concentración y de atención.

\section{Desarrollo del programa de intervención}

El programa de entrenamiento tiene una duración inicial de 5 meses, pudiéndose aplicar a 10 participantes como máximo a la vez, los cuales se le han diagnosticado EA en fase leve. Por falta de información previa, es necesario incluir medidas de control para evaluar los beneficios de esta intervención. En nuestro grupo de investigación, llevaremos a cabo el siguiente estudio piloto, para evaluar los beneficios de este diseño de intervención en personas con EA, el cual se desarrolla a continuación, incluyendo la descripción del programa de ejercicios a desarrollar.

\section{Proyecto de estudio piloto}

Todos los participantes del estudio formarán parte de las Asociaciones de Familiares Enfermos de Alzheimer (A.F.A.) de la Bahía de Cádiz, España. La muestra consistirá en 20 pacientes con diagnóstico de EA en su fase leve, divididos en dos grupos: (I) 10 sujetos en un grupo control (CG) que realizará las mismas actividades que el grupo experimental sin la práctica del surf; y (II) 10 participantes en un grupo de entrenamiento (TG) que participará en un programa de ejercicios multimodal, usando el surf como medio terapéutico. La edad media de los grupos estará comprendida entre los 50-65 años. Ambos grupos serán ajustados desde el principio por edad, educación, género, nivel de demencia, estado cognitivo global, nivel de capacidad aeróbica( $\mathrm{VO}_{2}$ máx.) y composición corporal.

Para ser finalmente incluidos en el programa, los pacientes con EA deben cumplir los siguientes criterios:

1. Diagnóstico neuropsicológico de EA en fase leve de la patología confirmado por el facultativo médico a partir de los resultados del Mini Mental State Examination (MMSE) (Blesa R. et al., 2001) y en acorde a los criterios de diagnóstico internacionales estandarizados (Huger, D. et al.; 2009).

2. Superación del examen médico pre-intervención en el que se declara que el enfermo no presentaba ninguna de las contraindicaciones incompatibles con el programa de ejercicios.

3. Obtención del consentimiento informado del interesado/a y/o cuidador/a.

Los criterios de exclusión serán los siguientes:

1. Presentar alguna de las enfermedades mentales siguientes: esquizofrenia, trastorno obsesivo compulsivo (TOC), o cualquier condición neuropsiquiátrica que pudiera interferir sobre la inter- vención o resultados, como por ejemplo la depresión o demenciamixta.

2. Modificar la prescripción de tratamiento durante la duración del estudio.

3. La presencia de cualquier patología que contraindique la práctica temporal o permanente del ejercicio físico, destacando como contraindicaciones absolutas la existencia de insuficiencia cardiaca en fase III o IV según la clasificación de la New York HearthAssociation (NYHA), la hipertensión arterial descompensada y el dolor agudo.

4. Drogodependencias.

5. La asistencia al entrenamiento debe ser continuada, los sujetos deben asistir al menos un $80 \%$ de las horas de sesión práctica. Si algún sujeto falta a más de 10 sesiones, quedará excluido del estudio.

6. Haber tenido experiencia previa con el surf.

\section{Objetivos de la intervención}

Desarrollarun programa de intervención con ejercicios capaces de mejorar el acondicionamiento físico, la capacidad cognoscitiva y el estado de salud de pacientes con EA en fase leve, a través de ejercicios multitarea y realizando un acercamiento al surf como medio terapéutico.

A través del programa de ejercicio físico, se plantea conseguir los siguientes subobjetivos:

- Mejorar la capacidad funcional y la condición física de los sujetos con EA en fase temprana, incrementando su autonomía o previniendo su deterioro.

- Mejorar los aspectos cognitivos: memoria, orientación espacial y temporal, fijación y concentración, afectados por la EA.

- Incrementar la capacidad de equilibrio para prevenir el riesgo de caída en personas mayores con EA a través de ejercicios funcionales adaptados a la población.

- Mejorar la calidad de vida de los sujetos con EA.

- Promover la actividad física en esta población específica de forma lúdica a través del surf como actividad saludable alternativa.

\section{Evaluación de las variables}

Después de realizar la anamnesis clínica con cada sujeto de la muestra seleccionada, se obtendrá información del historial clínico de los pacientes, identificando cualquier contraindicación del ejercicio. Se llevará a cabo una evaluación inicial usando el cuestionario de salud SF-36 para establecer un punto de partida respecto al estado del individuo, haciendo referencia a términos de la función física, social, mental y emocional, así como al estado de salud general y la calidad de vida (CdV). A partir de este cuestionario, se establece una serie de test para la valoración de la capacidad aeróbica, composición corporal, el equilibrio, la capacidad cognitiva y la autonomía en las actividades básicas de la vida diaria, que son los siguientes:

- Para la valoración de la condición física se llevará a cabo un test de esfuerzo en un ergonómetro modificado (Méndez, A. etal., 2005; Farkley, O. et al., 2012; Barlow, J., 2013) con un analizador de gases JaegerMasterScreen CPX ${ }^{\circledR}$ (CareFusion, San Diego, UnitedStates of America) [Figura 2] para determinar los siguientes valores: $\mathrm{VO}_{2}$ pico ( $\mathrm{VO}_{2}$ peak), ventilación por minuto (VE), umbral anaeróbico (UÂn) y

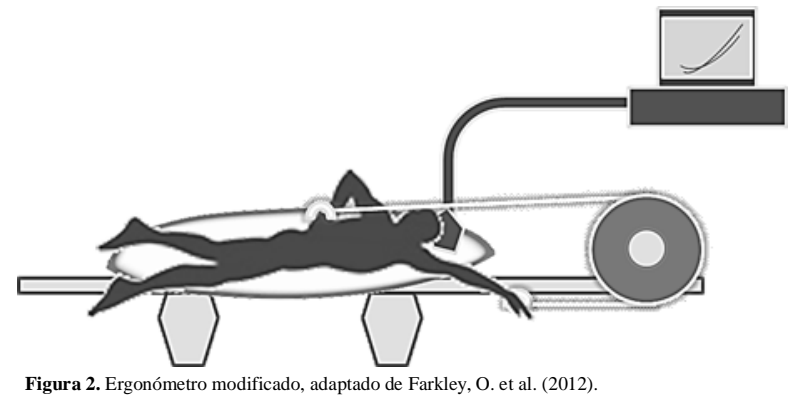

Figura 2. Ergonómetro modificado, adaptado de Farkley, O. et al. (2012).

Retos, número 32, 2017 ( $2^{\circ}$ semestre) 
aeróbico (UA), pulso de $\mathrm{O}_{2}$ y RER, FC pico (PeakHearthRate), potencia aeróbica pico (PeakAerobic Power), velocidad aeróbica pico (Peak Aerobic Speed), distancia total (Total Distance) y duración total (Total Duration).

- A su vez, se realizará un test de esfuerzo máximo en tapiz rodante, Treadmill Test (TT) con un analizador de gases JaegerMasterScreen CPX® (CareFusion, San Diego, UnitedStates of America) para determinar el $\mathrm{VO}_{2}$ pico como se ha descrito previamente (Ponce-González, JG. et al., 2015; Liu, R. et al., 2012; Vidoni, E. et al., 2012), siguiendo el protocolo de Bruce (Burns et al., 2008), que consta de 11 etapas, partiendo de una velocidad inicial fija de 2.735 km/h., sin inclinación. Cada dos minutos, la velocidad o grado de inclinación se aumentará de acuerdo con un protocolo diseñado para una población geriátrica (Hollenberg, M. et al., 1998). La escala de Borg[Rango 6-20] se utilizará para cuantificar el nivel de esfuerzo percibido por el sujeto en cada estadio de la prueba.

- La composición corporal se determinará por bioimpedancia y por mediciones antropométricas obteniendo el porcentaje de grasa y muscular (Camina, M.A., 2014).

- Se llevará a cabo la Escala de Berg (BBS), con un [Rango: 0-56], para la valoración del equilibrio en personas mayores, medido en base al rendimiento en la realización de tareas funcionales.

- Mini Mental State Examination (MMSE) adaptado por Blesa R. et al (2001)en versión español, con un [Rango: 0-30], para la valoración cognitiva de los sujetos. Y el ADAS test (Alzheimer DiseaseAssessment Scale), para observar el rendimiento cognitivo en pacientes con EA.

- La calidad de vida (CdV) en personas con Alzheimer se medirá a través del cuestionario validado QoL-AD, el cual consta de 13 ítems (Gómez-Gallego, M. et al., 2012). Por otro lado, se medirá con el índice de Barthel el grado de independencia a la hora de realizar actividades básicas de la vida diaria en diferentes parámetros, en un [Rango: 0-100]

Al final del protocolo de entrenamiento, se llevará a cabo una evaluación final durante la última sesión de trabajo del programa de entrenamiento, realizando el test de esfuerzo. Una semana después, se aplicará el resto de pruebas de valoración para observar comparativamente los resultados obtenidos respecto a los primeros test.

A continuación, se muestra un esquema de la temporalización del estudio [Figura 3], que incluye la programación temporal de los test que se llevarán a cabo:

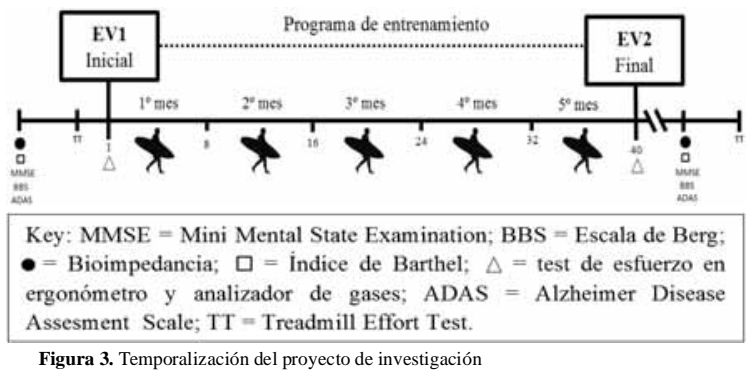

\section{Protocolo de entrenamiento}

El programa de intervención tendrá una duración de 5 meses tras el cual se llevará a cabo una evaluación para ambos grupos. Se realizará dos sesiones semanales de 90 minutos de trabajo en grupo con ejercicios multimodales centrados en el trabajo de equilibrio y las actividades de doble tarea, combinado con surf como medio terapéutico en el grupo de entrenamiento (TG), mientras que el grupo control (CG) realizará las mismas actividades que el grupo de intervención, salvo la actividad del surf.

El objetivo del programa de entrenamiento es trabajar las dimensiones motriz, cognitiva, afectiva y social de los sujetos. Por este motivo, centramos su diseño en tres aspectos importante para los sujetos con EA: el ejercicio físico, la estimulación cognitiva y la estimulación psicomotriz (Serdá I Ferrer, B. et al., 2013). Los sujetos trabajarán de manera individualizada con cada monitor, aunque la sesión se organiza grupalmente.
Las sesiones se dividirán en cuatro partes, que se llevarán a cabo de manera grupal:

(I) Calentamiento: incluirá ejercicios de movilidad articular y aeróbicos generales, favoreciendo el aumento de la temperatura y la adaptación del organismo al esfuerzo posterior;

(II) Ejercicios funcionales adaptados en circuito, con transferencia positiva con la práctica del surf, basándonos en dos pilares básicos: (II.1)Actividades de doble tarea, realizando ejercicios donde se combina acciones simples con la estimulación cognitiva del individuo, trabajando la memoria, la orientación, la fijación, la concentración y el lenguaje. (II.2) Ejercicios de acondicionamiento físico, donde se trabajará la capacidad aeróbica, la propiocepción, la flexibilidad, la fuerza y la agilidad; además se realizarán actividades para el desarrollo del equilibrio (fundamental para prevenir el riesgo de caídas en personas mayores).

(III) Práctica del surf como medio terapéutico: se realizarán ejercicios de remada y equilibrio dinámico en el agua. Los sujetos trabajan de manera individualizada con un monitor, que los ayudará en sus tareas, corrigiendo la posición y concienciándolos del control postural. Además, los lanzará para facilitar que remen la ola, y controlarán el momento del take off para asegurar que tengan éxito en sus acciones.

(IV) Vuelta a la calma: recordatorio de los momentos de la sesión y las sesiones anteriores, para estimular el área cognitiva del cerebro. Comentario grupal fomentando la cohesión entre los individuos y ejercicios de vuelta a la calma para volver a los niveles basales.

\section{Análisis estadístico}

Se aplicará el test de Shapiro-Wilks para determinar si cumple las variables dependientes una distribución normal. Los resultados se expresarán como media \pm desviación estándar(SD). Las posibles relaciones entre variables se determinará mediante correlación lineal bivariada (coeficiente de Pearson). Para determinar las diferencias transversales se usará la prueba t-student de muestras independientes, y para determinar las diferencias longitudinales la prueba t-student de muestras relacionadas. Análisis multifactorial de la varianzaANOVA se usará para el análisis longitudinal con posibles cofactores, aplicando la prueba post hoc por Bonferroni. El análisis de los datos se considera significativo estadísticamente cuando $\mathrm{P}<0.05$. Los datos serán analizados usando el programa estadístico SPSS19.0. (SPCC Inc., USA).

\section{Resultados esperados}

En función de los progresos hallados basándonos en evidencias científicas; con la realización del programa de intervención, se espera obtener los siguientes resultados sobre las variables estudiadas. Los datos muestran aquellos factores que guardan relación, principalmente, con la enfermedad deAlzheimer y el deterioro de la capacidad cognitiva y funcional de los sujetos.

Se espera una mejora significativa en las diferentes dimensiones relacionadas con el rendimiento cognitivo, principalmente en la orientación, la concentración y la memoria. Reduciendo el impacto del deterioro cognitivo.

Se estima una mejora en las pruebas de equilibrio, reduciendo de tal manera, el riesgo de sufrir caídas, y mostrando un mejor desempeño en sus actividades de la vida diaria.

Además de la mejora significativa de los valores relacionados con la enfermedad de Alzheimer obtenidos en los diferentes test; se espera una mejora considerable, al final del plan de entrenamiento, de los parámetros de condición física y autonomía personal, respecto a la prueba de esfuerzo realizada inicialmente.

\section{Conclusiones}

Estimamos que la intervención planteada usando el surf como herramienta terapéutica puede provocar el estímulo necesario para retrasar los efectos de la enfermedad del Alzheimer sobre el organismo teniendo en cuenta los resultados de nuestra revisión sobre el ejercicio y 
la hidroterapia en este tipo de personas. El surf puede mejorar la condición física tal y cómo se describe en todos los artículos analizados, este aspecto resulta ser crucial en la mejora de las personas con EA tras un programa de ejercicio. Los principales problemas a los que nos afrontamos con este tipo de intervención son la dificultad de la tarea y el nivel heterogéneo que nos podemos encontrar en la población seleccionada, sin embargo, la intervención va orientada a realizar surf recreacional, el cual puede realizar cualquier persona, incluso con ciertas patologías como se ha demostrado en diferentes asociaciones con personas con síndrome de Down o autismo.

\section{Referencias}

Armitano, C. (2013). Effectiveness of a Surfing Intervention on Children with Disabilities. Open Access Master's Theses. Paper 13.

Baker, L. D. L., \& Frank, L. (2010). Effects of Aerobic Exercise on Mild Cognitive Impairment: a controlled trial. Archives of Neurology, 67(1), 71-79.

Barlow, M. J. (2013). The Effects of Anthropometrical, Physiological and Environmental Factors on Surfing Performance. School of Marine Science and Engineering. Plymouth University.

Blesa R, Pujol M, Aguilar M, Santacruz P, Bertran-Serra I, Hernandez G ... \& NORMACODEM Group (2001). Clinical validity of the 'mini-mental state' for Spanish speaking communities. Neuropsychologia, 39 (11), 1150-1157.

Burge, E., Kuhne, N., Berchtold, A., Maupetit, C., \& von Gunten, A. (2012) Impact of physical activity on activity of daily living in moderate to severe dementia: a critical review. European Review of Aging and Physical Activity, 9(1), 27-39.

Burns, J. M., Cronk, B. B., Anderson, H. S., Donnelly, J. E., Thomas, G. P., Harsha, A., ... \& Swerdlow, R. H. (2008). Cardiorespiratory fitness and brain atrophy in early Alzheimer disease. Neurology, 71(3), 210-216.

Camina Martín, M.A.(2014) Aplicaciones del análisis de bioimpedancia en la valoración del estado nutricional en ancianos institucionalizados con demencia. Universidad de Valladolid, Facultad de Medicina.

Canonici, A. P., Andrade, L. P. De, Gobbi, S., Santos-Galduroz, R. F., Gobbi, L. T. B., \& Stella, F. (2012). Functional dependence and caregiver burden in Alzheimer's disease: A controlled trial on the benefits of motor intervention. Psychogeriatrics, 12(3), 186-192.

Chrysagis, N., Douka, A., Nikopoulos, M., Apostolopoulou, F., \& Koutsouki, D. (2009). Effects of an aquatic program on gross motor function of children with spastic cerebral palsy. Journal of Biology of Exercise, 5, 13-25.

Clapham, E D., Armitano, C. N., Lamont, L. S., \&Audette, J. G. (2014). «The Ocean as a Unique Therapeutic Environment/: Developing a Surfing Program», Journal of Physical Education, Recreation \& Dance, 85 (4), 8-14

Cohelo, F. G. D. M., Andrade, L. P., Pedroso, R. V., Santos-Galduroz, R. F., Gobbi, S., Costa, J.L. R., \& Gobbi, L. T.B. (2013). Multimodal exercise intervention improves frontal cognitive functions and gait in Alzheimer's disease: Acontrolled trial. Geriatrics and Gerontology International, 13(1), 198-203.

Conatser, P. (2007). Adapted Aquatics \& Rehabilitation/ : A Literature Synthesis. International Journal of Aquatic Research and Education, 1, 242-254.

DeAndrade, L. P., Gobbi, L. T. B., Coelho, F. G, Christofoletti, G.,Costa, J. L., \& Stella, F. (2013). Benefits of multimodal exercise intervention for postural control and frontal cognitive functions in individuals with Alzheimer's disease: a controlled trial. Journal of the American Geriatrics Society, 61(11), 1919-1926.

EFE (15 de Septiembre de 2011)Los cuidados del Alzheimer en España suponen 24.000 millones de euros anuales. El País. Madrid (España).

Fang, Y., \& Swartwood, R. M. (2012). Feasibility and Perception of the Impact From Aerobic Exercise in Older Adults With Alzheimer's
Disease. American Journal of Alzheimer's Disease and Other Dementias, 27(6), 397-405.

Farley, O., Harris, N. K., \& Kilding, A. E. (2012). Anaerobic and aerobic fitness profiling of competitive surfers. Journal of Strength \& Conditioning Research, 26(8), 2243-2248.

Ferrer, S. I., Ortiz Collado, A., \& Àvila-Castells, P. (2013). Reeducación psicomotriz en personas con enfermedad de Alzheimer. Revista médica de Chile, 141(6), 735-742.

Fleischmann, D., Michalewicz, B., Stedje-Larsen, E., Neff, J., Murphy, J., Browning, K., ... \& Herriman, L. (2011). Surf Medicine: Surfing as a Means of Therapy for Combat-Related Polytrauma. JPO: Journal of Prosthetics and Orthotics, 23(1), 27-29.

Garuffi, M., Costa, J. L. R., Hernández, S. S. S., Vital, T. M., Stein, A. M., Santos, J. G. D., \& Stella, F. (2013). Effects of resistance training on the performance of activities of daily living in patients with Alzheimer's disease. Geriatrics \& gerontology international, 13(2), 322-328.

Gómez-Gallego, M., Gómez-Amor, J., \& Gómez-García, J. (2012). Validación de la versión española de la escala QoL-AD en pacientes con enfermedad de Alzheimer, cuidadores y profesionales sanitarios. Neurología, 27(1), 4-10.

Hauer, K., Hüger, D., Zieschang, T., Schwenk, M., Oster, P., Becker, C., \& Hauer, K. (2009). Designing studies on the effectiveness of physical training in patients with cognitive impairment. Zeitschrift für Gerontologie und Geriatrie, 42(1), 11-19.

Hernandez, S. S., Coelho, F. G, Gobbi, S., \& Stella, F. (2010). Effects of physical activity on cognitive functions, balance and risk of falls in elderly patients with Alzheimer's dementia. Revista Brasileira de Fisioterapia, 14(1), 68-74.

Hollenberg, M., Ngo, L. H., Turner, D., \& Tager, I. B. (1998). Treadmill exercise testing in an epidemiologic study of elderly subjects. The Journals of Gerontology Series A: Biological Sciences and Medical Sciences, 53(4), B259-B267.

Liu, R., Sui, X., Laditka, J. N., Church, T. S., Colabianchi, N., Hussey, J., \& Blair, S. N. (2012). Cardiorespiratory fitness as a predictor of dementia mortality in men and women. Medicine and science in sports and exercise, 44(2), 253-259

Maniu, D. A., Maniu, E. A., \& Benga, I. (2013). Effects of an aquatic therapy program on vital capacity , quality of life and physical activity index in children with cerebral palsy. International Journal of the Bioflux Society, 5(3), 117-124.

Meier, R. A., Lowdon, B. J., \& Davie, A. J. (1991). Heart Rates and Estimated Energy Expenditure During Recreational Surfing.The Australian Journal of Science and Medicine in Sport, 23(3), 70-74.

Mendez-Villanueva, A., \& Bishop, D. (2005). Physiological aspects of surfboard riding performance. Sports Medicine, 35(1), 55-70.

Montón, F., Rodriguez, N., Ruíz, N., Rojo, J.A., Bueno, J., Pérez, D, ..., Gómez, M. (2011). Manual de Actuación en la enfermedad de Alzheimer y otras Demencias. Servicio Canario de Salud, Gobierno de Canarias.

Ponce-González JG, Olmedillas H, Calleja-González J, Guerra B, Sanchis-Moysi J. (2015). Physical fitness, adiposity and testosterone concentrations are associated to playing position in professional basketballers. Nutrición Hospitalaria, 31(6):26242632.

Stella, F., Canonici, A. P., Gobbi, S., Galduroz, R. F. S., Cação, J. D. C., \& Gobbi, L.T.B.(2011). Attenuation of neuropsychiatric symptoms and caregiver burden in Alzheimer's disease by motor intervention: a controlled trial. Clinics, 66(8), 1353-1360.

Vidoni, E. D., Honea, R. A., Billinger, S. A., Swerdlow, R. H., \& Burns, J. M. (2012). Cardiorespiratory fitness is associated with atrophy in Alzheimer's and aging over 2 years. Neurobiology of Aging, 33(8), 1624-1632.

Zhou, S., Martin, F., Bezerra, P. \& Crowley, Z. (2008). An investigation on the effects of recreational surfing on control of force and posture in older surfers. International Convention on Science, Education and Medicine in Sport, 3, 336. 